\title{
Descending Aorta Blood Flow Characteristics before the Development of Necrotizing Enterocolitis in Preterm Neonates
}

Kyung Min Kim, Hyo Sup Kim, Ji Hong Yoon, Eun-Jung Lee, Sook Kyung Yum, Cheong-Jun Moon, Young-Ah Youn, Yoo Jin Kwun, Jae Young Lee, and In Kyung Sung

Department of Pediatrics, Seoul St. Mary's Hospital, College of Medicine, the Catholic University of Korea, Seoul, Korea

\section{ABSTRACT}

Purpose: To investigate the hemodynamic risk factors for necrotizing enterocolitis (NEC), we analyzed the characteristics of descending aorta (DA) blood flow in preterm neonates, who later developed NEC.

Methods: This was an observational case-control study on 53 preterm neonates at a tertiary referral center. Clinical and echocardiographic data were collected from 23 preterm neonates with NEC (NEC group), and compared with those of 30 preterm neonates without NEC (control group). Echocardiography was done at a median (interquartile range) of 5 (3-9) days after birth and 2 (1-2.5) days before the diagnosis of NEC.

Results: Basic clinical characteristics including gestational age, birth weight, Apgar score, breast feeding status, use of umbilical catheters, and mode of invasive ventilator care were similar between the groups. Compared with the control group, the lowest diastolic velocity of DA was significantly decreased, whereas the diastolic reverse flow and the ratio of diastolic reverse to systolic forward flows were significantly increased in the NEC group. In addition, the resistive index (RI) of DA was significantly increased in the NEC group and showed a positive association with the development of NEC. Multivariate logistic regression analysis showed that increasing RI of DA was an independent risk factor for the development of $\operatorname{NEC}(P=0.008)$.

Conclusion: Significant changes in DA flow characteristics including decreased diastolic velocity and increased diastolic reverse flow along with increased peripheral vascular resistance were observed before the development of NEC in preterm neonates. These findings may help clinicians stratify in advance neonates at a risk of developing NEC and may help improve outcomes in these neonates.

Key Words: Doppler flow study, Critical care, Splanchnic circulation, Vascular resistance, Necrotizing enterocolitis, Preterm neonates
Received: 24 September 2017

Revised: 7 March 2018

Accepted: 26 April 2018

Correspondence to: Jae Young Lee Department of Pediatrics, Seoul St. Mary's Hospital, College of Medcine, the Catholic University of Korea, 222 Banpo-daero, Seocho-gu, Seoul 06591, Korea

Tel: +82-2-2588-6189

Fax: +82-2-537-4544

E-mail: jaeyounglee@catholic.ac.kr

Copyright(c)

By Korean Society of Neonatology.

All right reserved.

This is an Open-Access article distributed under the terms of the Creative Commons Attribution Non-Commercial License (http://creativecommons.org/licenses/ by-nc/4.0), which permits unrestricted non-commercial use, distribution, and reproduction in any medium, provided the original work is properly cited. 


\section{INTRODUCTION}

Necrotizing enterocolitis (NEC) is an important cause of morbidities and mortality in preterm neonates. Owing to the multifactorial etiology of NEC and an unclear diagnosis, proper timely management is sometimes delayed, leading to surgical intervention and associated complications, with mortality in most of the severe cases ${ }^{1-3)}$. Given that the development of NEC is unexpected, its progression is rapid, and the condition is associated with poor outcomes, it is important to stratify in advance those high risk neonates who may have NEC.

Of the several risk factors attributed to the development of NEC, current studies are focusing on the potential role of gut ischemia and injury with subsequent inflammatory reactions of the intestinal wall ${ }^{4-8)}$. Furthermore, ischemia has been suggested as an important factor in the development of "fulminant" $\mathrm{NEC}^{5,9)}$. In this regard, the blood flow patterns of the superior mesenteric artery (SMA) were previously studied using noninvasive ultrasound methods to investigate their associations with the development of NEC in preterm neonates ${ }^{10-13)}$. The studies showed that an increased resistance pattern of blood flow velocity in the SMA was an independent risk factor for the developing NEC. On the other hand, for term infants with congenital heart diseases, an altered flow pattern of the descending aorta (DA) was associated with the development of $\mathrm{NEC}^{14,15)}$. However, the descending aorta blood flow (DABF) characteristics have not been studied in preterm neonates with NEC.

In this study, we aimed to analyze the characteristics of DABF by echocardiographic spectral Doppler imaging in preterm neonates with NEC, and to compare the findings with those of preterm neonates without NEC as a control group. We hypothesized that altered blood flow characteristics of the DA may have an influence on the splanchnic circulation, thereby affecting the development of NEC in preterm neonates, and that early detection of such altered blood flow characteristics may help clinicians stratify in advance those preterm neonates at a risk of developing NEC.

\section{MATERIALS AND METHODS}

\section{Study design}

This study was an observational case-control study on 23 preterm neonates with NEC (NEC group), and 30 preterm neo- nates without NEC (control group) at a tertiary referral neonatal intensive care unit between July 2014 and October 2016. The Institutional Review Board of Seoul St. Mary's Hospital approved this study and waived to obtain informed consent.

NEC was diagnosed on the basis of the patient's systemic, intestinal, and radiologic signs and staged according to the modified Bell's classification ${ }^{1,16)}$. Preterm neonates who had been managed under the diagnosis of NEC (NEC stage $\geq 1$ ) and had undergone a comprehensive echocardiographic evluation before 14 days of age and within 7 days before the diagnosis of NEC comprised NEC group $(n=23)$. The control group $(n=30)$ consisted of preterm neonates who received neonatal intensive care during the same period but did not have NEC and echocardiographic study before 14 days of age approximated to the day of life of their NEC counterparts. Neonates who had structural heart diseases, structural anomalies in the gastrointestinal tract or culture-proven sepsis were excluded from the study. Basic clinical characteristics

Table 1. Basic Clinical Characteristic of the Study Population

\begin{tabular}{lcc}
\hline Variable & $\begin{array}{c}\text { NEC group } \\
(\mathrm{n}=23)\end{array}$ & $\begin{array}{c}\text { Control group } \\
(\mathrm{n}=30)\end{array}$ \\
\hline Female sex & $10(43)$ & $9(30)$ \\
Gestational age (wk) & $28.2 \pm 3.1$ & $27.9 \pm 2.7$ \\
Birth weight (kg) & $1.03 \pm 0.30$ & $1.14 \pm 0.38$ \\
Apgar score & & \\
1 min & $2.6 \pm 1.8$ & $2.8 \pm 1.9$ \\
5 min & $5.6 \pm 2.1$ & $5.4 \pm 2.1$ \\
Cesarean delivery & $20(87)$ & $28(93)$ \\
Breast feeding & $7(30)$ & $8(27)$ \\
Umbilical venous catheter & $23(100)$ & $26(87)$ \\
Umbilical arterial catheter & $5(22)$ & $8(27)$ \\
Ventilator care* & & \\
Conventional mode & $10(43)$ & $12(40)$ \\
HFOV mode & $8(35)$ & $12(40)$ \\
Age at echocardiogram (day) & $5(3-9)$ & $4(3-8)$ \\
Age at NEC diagnosis (day) & $8(5-11)$ & - \\
Interval of echo-NEC diagnosis (day) & $2(1-2.5)$ & - \\
NEC stage & & - \\
Stage 1 & $12(52)$ & - \\
Stage 2 & $5(22)$ & - \\
Stage 3 & $6(26)$ & - \\
\hline
\end{tabular}

Values are expressed as number (\%), mean \pm standard deviation, or median (interquartile range). There were no significant differences in variables between the NEC and control groups.

*Invasive ventilator care when echocardiogram was performed. Abbreviations: NEC, necrotizing enterocolitis; HFOV, high frequency oscillation ventilation. 
and echocardiographic hemodynamic data were collected from our institutional database, and compared between the NEC and control groups. The NEC group was further classified into three subgroups according to the NEC stages, and echocardiographic

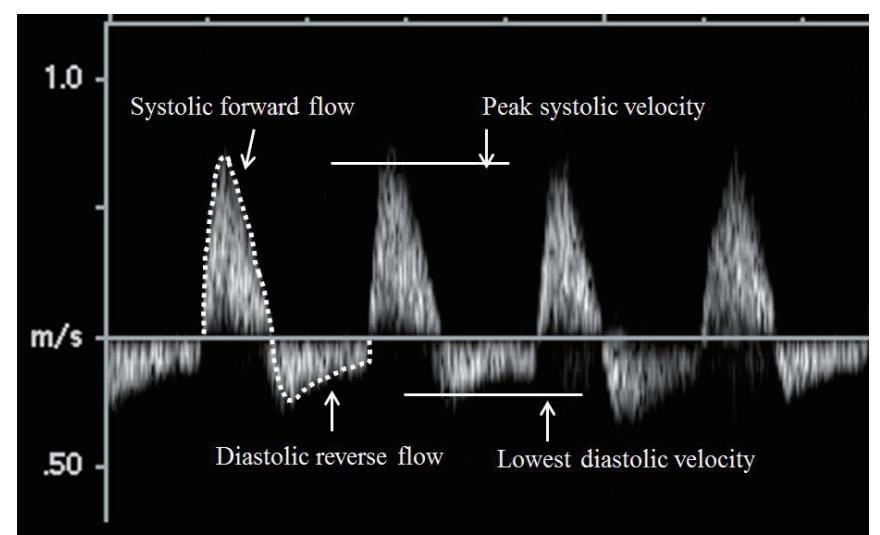

Figure 1. A spectral Doppler image of descending aorta flow showing a systolic forward flow and a diastolic reverse flow. Descending aorta blood flow and diastolic reverse flow were calculated using the velocity time integral of the systolic forward flow and diastolic reverse flow, respectively (dotted lines). A resistive index (RI) was calculated from peak systolic velocity and lowest diastolic velocity. $\mathrm{RI}=$ (peak systolic velocity-lowest diastolic velocity)/peak systolic velocity. hemodynamic data were compared among the subgroups.

\section{Echocardiographic hemodynamic assessment}

Echocardiographic hemodynamic assessments were done by an experienced pediatric cardiologist according to the protocol of our pediatric cardiology program, and all images were checked for appropriate quality required for analyses by an experienced consultant pediatric cardiologist, as previously reported ${ }^{17)}$. In summary, for conventional assessment, the left ventricular ejection fraction (LVEF), and left ventricular fractional shortening (LVFS) were measured from the parasternal long-axis view. From the spectral pulsed-wave Doppler of the DA, the resistive index (RI), a surrogate of peripheral vascular resistance, was calculated, as follows: $\mathrm{RI}=$ (peak systolic velocity-lowest diastolic velocity)/ peak systolic velocity (Figure 1$)^{18)}$. The DABF was derived by multiplying, the cross-sectional area of the DA, the velocity time integral (VTI) of the DA Doppler at the level of the diaphragm in a low subcostal sagittal view, and the heart rate ${ }^{17)}$. The diastolic reverse flow of the DA, if any, was calculated by multiplying the VTI of diastolic reverse flow, the cross-sectional area of the DA, and the heart rate (Figure 1). The ratio of the diastolic reverse flow of DA to the DABF was also derived. To obtain pulsed-

Table 2. Echocardiographic Hemodynamic Characteristics

\begin{tabular}{|c|c|c|c|}
\hline Variable & $\begin{array}{l}\text { NEC group } \\
(n=23)\end{array}$ & $\begin{array}{l}\text { Control group } \\
\qquad(\mathrm{n}=\mathbf{3 0})\end{array}$ & $P$-value \\
\hline Heart rate (beats/min) & $154 \pm 15$ & $154 \pm 17$ & NS \\
\hline Diastolic BP (mm Hg) & $32.7 \pm 8.3$ & $32.5 \pm 7.4$ & NS \\
\hline $\operatorname{LVEF}(\%)$ & $61.6 \pm 5.6$ & $61.9 \pm 8.0$ & NS \\
\hline LVFS (\%) & $38.2 \pm 4.7$ & $38.7 \pm 7.1$ & NS \\
\hline Lowest diastolic velocity of DA $(\mathrm{cm} / \mathrm{sec})$ & $-8.4(-24.1$ to 4.6$)$ & $0(-14.8$ to 10.7$)$ & $<0.001$ \\
\hline Resistive index & $1.21 \pm 0.21$ & $0.98 \pm 0.15$ & $<0.001$ \\
\hline $\mathrm{DABF}(\mathrm{mL} / \mathrm{kg} / \mathrm{min})$ & $104.6 \pm 45.9$ & $128.1 \pm 47.5$ & 0.06 \\
\hline Diastolic reverse flow of DA $(\mathrm{mL} / \mathrm{kg} / \mathrm{min})$ & $12.9(0$ to 101.8$)$ & $0(0$ to 68.4$)$ & 0.007 \\
\hline Diastolic reverse flow of DA/DABF (\%) & $20(0$ to 62$)$ & $0(0$ to 36$)$ & 0.002 \\
\hline Left atrium to aortic root ratio & $1.29 \pm 0.24$ & $1.38 \pm 0.22$ & NS \\
\hline Peak transductal flow velocity (m/sec) & $1.35 \pm 0.53$ & $1.27 \pm 0.62$ & NS \\
\hline HSDA & $4(17)$ & $5(17)$ & NS \\
\hline
\end{tabular}

Values are expressed as mean \pm standard deviation, median (range), or number (\%).

Abbreviations: NEC, necrotizing enterocolitis; NS, not significant; BP, blood pressure; LVEF, left ventricular ejection fraction; LVFS, left ventricular fractional shortening; DA, descending aorta; DABF, descending aorta blood flow; HSDA, hemodynamically significant ductus arteriosus. 
wave Doppler images of the DA for calculating the DABF and RI, an appropriate Doppler angle correction was applied. In case of ductus arteriosus, its diameter and transductal Doppler velocity were measured in a high parasternal 'ductal' view. The dimensions of left atrium and aortic root were measured in the parasternal long-axis view. An echocardiographic hemodynamically significant ductus arteriosus (HSDA) was defined as the presence of ductus arteriosus $\geq 1.5 \mathrm{~mm}$ with an unrestrictive pulsatile transductal flow (peak Doppler velocity $<2.0 \mathrm{~m} / \mathrm{sec}$ ) and a left atrium to aortic ratio of $\geq 1.5$. All echocardiographic flow indices were recorded from the average value of three consecutive cardiac cycles.

\section{Statistical analysis}

Data were analyzed using commercially available software (PASW statistics version 18 for Window; IBM, Armonk, NY, USA). Data were expressed as mean \pm standard deviation, median (interquartile range [IQR]), or number (\%). Comparisons of basic clinical characteristics and echocardiographic parameters between the NEC and control groups were conducted using a 2-tailed, independent-samples $t$-test for normally distributed data and a 2-tailed Mann-Whitney $U$-test for data that were not normally distributed, or Fisher exact test when appropriate. Comparison of echocardiographic parameters among the three NEC subgroups and the control group were performed using the Kruskal-Wallis test. Univariate and multivariate logistic regression analyses were done to identify the risk factors for NEC development. A $P$-value less than 0.05 was considered statistically significant.

\section{RESULTS}

\section{Basic clinical characteristics}

A total of 53 preterm neonates were enrolled, including 23 neonates who developed NEC (NEC group) and 30 neonates without NEC (control group) during the study period. The basic clinical characteristics of each group are summarized in Table 1. The two groups had similar basic clinical characteristics with regard to gestational age, birth weight, Apgar score, mode of delivery, mode of feeding, types of central venous catheters and the ratio and mode of invasive ventilator care. In the NEC group, NEC was diagnosed at a median of 8 days (IQR, 5 to 11) after birth. There were 12 neonates (52\%) with stage 1 NEC, five neonates (22\%) with stage 2 NEC, and six neonates (26\%) with stage 3 NEC according to the modified Bell's classification. Echocardiographic evaluation were done at a median 5 days (IQR, 3 to 9) for the NEC group, and 4 days (IQR, 3 to 8) for the control group after birth, respectively ( $P=$ not significant). In the NEC group, echocardiography was done at a median 2 (IQR, 1 to 2.5) before the diagnosis of NEC.

\section{Echocardiographic hemodynamic characteristics}

The echocardiographic hemodynamic characteristics of each group are summarized in Table 2. Compared with the control group, the NEC group had significantly decreased Doppler diastolic velocity, and significantly increased RI, diastolic reverse flow of the DA and the ratio of diastolic reverse flow of the DA to the DABF. Other parameters including blood pressure, LVEF, LVFS, and peak systolic velocity of the DA were similar between the groups. The incidence of ductus arteriosus, its diameter, left atrium to aortic ratio, and incidence of HSDA were also similar between the groups.

The subgroup analysis in the NEC group according to the NEC stages showed significant differences in the peak systolic velocity, lowest diastolic velocity, RI, DABF, diastolic reverse flow of the DA and the ratio of diastolic reverse flow of the DA to DABF among the subgroups (Table 3). The higher the NEC stage, the more significant decrease in the peak systolic velocity, lowest diastolic velocity and DABF, whereas more significant increase in the RI, diastolic reverse flow of DA, and the ratio of diastolic reverse flow of DA to DABF were noted. The proportions of patients with HSDA were similar among NEC subgroups.

To identify the risk factors for the development of NEC, logistic regression analyses were done for echocardiographic hemodynamic parameters (Table 4). In univariate analysis, the lowest diastolic velocity of the DA, RI, diastolic reverse flow of the DA and the ratio of diastolic reverse flow of the DA to the DABF were associated with the development of NEC. In multivariate analysis, the increasing RI of DA flow was an independent risk factor for development of NEC $(P=0.008)$.

\section{DISCUSSION}

The key finding of this study was that altered blood flow characteristics of the DA were associated with the development of NEC in preterm neonates. Neonates who developed NEC later had significantly decreased diastolic velocity, significantly 
Table 3. Echocardiographic Hemodynamic Characteristics of the Control Group and NEC Subgroups According to NEC Stages

\begin{tabular}{|c|c|c|c|c|c|}
\hline \multirow{2}{*}{ Characteristic } & \multirow{2}{*}{$\begin{array}{l}\text { Control group } \\
\qquad(\mathrm{n}=\mathbf{3 0})\end{array}$} & \multicolumn{3}{|c|}{ NEC group $(n=23)$} & \multirow{2}{*}{$P$-value } \\
\hline & & Stage $1(n=12)$ & Stage $2(n=5)$ & Stage $3(n=6)$ & \\
\hline Heart rate (beats/min) & $154 \pm 17$ & $149 \pm 15$ & $166 \pm 11$ & $152 \pm 17$ & NS \\
\hline Diastolic BP (mm Hg) & $32.5 \pm 7.4$ & $30.5 \pm 6.0$ & $41.4 \pm 11.8$ & $29.7 \pm 3.1$ & NS \\
\hline $\operatorname{LVEF}(\%)$ & $61.9 \pm 8.0$ & $62.8 \pm 6.2$ & $57.9 \pm 2.3$ & $62.1 \pm 5.6$ & NS \\
\hline Lowest diastolic velocity of DA $(\mathrm{cm} / \mathrm{sec})$ & $0(-14.8$ to 10.7$)$ & $0(-24.2$ to 4.6$)$ & $-8.4(-17.4$ to 0$)$ & $-9.8(-17.0$ to 0$)$ & 0.003 \\
\hline Resistive index & $0.98 \pm 0.15$ & $1.1 \pm 0.2$ & $1.2 \pm 0.2$ & $1.4 \pm 0.2$ & 0.001 \\
\hline $\mathrm{DABF}(\mathrm{mL} / \mathrm{kg} / \mathrm{min})$ & $128.1 \pm 47.5$ & $116.5 \pm 48.6$ & $95.2 \pm 34.4$ & $75.2 \pm 45.9$ & 0.001 \\
\hline Diastolic reverse flow of DA (mL/kg/min) & $0(0$ to 68.4$)$ & $0(0$ to 101.8$)$ & $40.2(0$ to 77.4$)$ & $17.3(0$ to 58.6$)$ & 0.01 \\
\hline Ductus arteriosus $>1.5 \mathrm{~mm}$ & $18(60)$ & $8(67)$ & $4(80)$ & $6(100)$ & NS \\
\hline Left atrium to aortic root ratio & $1.38 \pm 0.22$ & $1.30 \pm 0.17$ & $1.33 \pm 0.32$ & $1.27 \pm 0.31$ & NS \\
\hline Peak transductal flow velocity (m/sec) & $1.27 \pm 0.62$ & $1.43 \pm 0.67$ & $1.35 \pm 0.60$ & $1.25 \pm 0.31$ & NS \\
\hline HSDA & $5(17)$ & $2(17)$ & $1(20)$ & $1(17)$ & NS \\
\hline
\end{tabular}

Values are expressed as mean \pm standard deviation, median (range), or number (\%).

Abbreviations: NEC, necrotizing enterocolitis; NS, not significant; BP, blood pressure; LVEF, left ventricular ejection fraction; LVFS, left ventricular fractional shortening; DA, descending aorta; DABF, descending aorta blood flow; HSDA, hemodynamically significant ductus arteriosus.

Table 4. Logistic Regression Analysis of Echocardiographic Parameters Predicting the Risk of Developing Necrotizing Enterocolitis

\begin{tabular}{lccc}
\hline Variable & OR & $95 \%$ CI & $P$-value \\
\hline Systolic BP & 1.00 & $0.95-1.06$ & NS \\
Diastolic BP & 1.00 & $0.93-1.08$ & NS \\
LVEF & 0.99 & $0.92-1.08$ & NS \\
LVFS & 0.96 & $0.91-1.02$ & NS \\
Peak systolic velocity & 0.95 & $0.91-1.00$ & NS \\
Lowest diastolic velocity & 0.88 & $0.81-0.95$ & 0.002 \\
Resistive index $(\times 10)$ & 2.00 & $1.36-2.94$ & 0.001 \\
DABF & 0.99 & $0.98-1.00$ & NS \\
Diastolic reverse flow of DA & 1.03 & $1.00-1.06$ & 0.02 \\
Diastolic reverse flow of DA/DABF & 1.06 & $1.02-1.09$ & 0.004 \\
\hline
\end{tabular}

Abbreviations: OR, odds ratio; CI, confidence interval; BP, blood pressure; NS, not significant; LVEF, left ventricular ejection fraction; LVFS, left ventricular fractional shortening; DABF, descending aorta blood flow; DA, descending aorta.

increased resistance patterns of blood flow velocity, and prominent diastolic reverse flow in the DA. Increasing RI of the DA was an independent risk factor for NEC development. In addition, the RI values were significantly different among NEC subgroups according to the NEC stages.
Of the multiple pathologic conditions and precipitating factors considered to contribute to the development of NEC, mucosal ischemia and decreased oxygen delivery to the gastrointestinal tract caused by an altered splanchnic circulation and subsequent inflammatory reactions are suggested as key precipitating factors in the pathophysiology of $\mathrm{NEC}^{5-7,10)}$. To assess the splanchnic circulation noninvasively, the flow characteristics of the SMA, as determined using ultrasound spectral Doppler velocimetry have been investigated in neonates with $\mathrm{NEC}^{10-12)}$. Hashem et al. ${ }^{10)}$ reported that septic neonates with NEC had a significantly lower peak systolic velocity and a lower end-diastolic velocity in the SMA than septic neonates without clinical signs of NEC. Murdoch et al. ${ }^{11)}$ reported that neonates with high resistance patterns (high pulsatility index) of blood flow velocity in the SMA on the first day of life are at an increased risk of developing NEC. Urboniene et al. ${ }^{12)}$ measured the RI and pulsatility index of the SMA in 62 neonates (29 neonates with NEC and 33 neonates as the control group), and found that $96.3 \%$ of the neonates with NEC had RI $>0.75$ with a sensitivity of $96.3 \%$ and a specificity of $90.9 \%$ (odds ratio $[\mathrm{OR}] 26$ ), and $88.9 \%$ of the neonates with NEC had a pulsatility index $>1.85$ with a sensitivity of $88.9 \%$ and a specificity of $78.8 \%$ (OR, 29). The authors suggested that blood 
flow Doppler velocimetry of the SMA could be a useful tool for diagnosing and predicting $\mathrm{NEC}^{10,12)}$.

For assessing the splanchnic circulation, Doppler flow characteristics of the DA in preterm neonates with NEC have not been reported. Only a few studies in term neonates with congenital heart disease showed that decreased peak systolic forward velocity and persistent diastolic flow reversal in the abdominal aorta were associated with an increased risk of $\mathrm{NEC}^{14,15)}$. In this study, we investigated the Doppler flow characteristics of the DA in preterm neonates with NEC, assuming that altered blood flow patterns of the DA may have an influence on the splanchnic circulation, and thereby affect the development of NEC. In clinical practice, Doppler velocimetry measurement of the SMA is seldom feasible owing to the poor window due to gaseous distension of the abdomen and the small vessel diameter of the SMA in premature babies. However, from our experience, measurement of the DA flow was easier and much more feasible than measurement of the SMA flow.

In our study, decreased diastolic velocity and increased reverse flow and RI, indicating decreased perfusion to the lower body including the splanchnic circulation, were associated with the development of NEC in preterm neonates. These findings were consistent with those of previous studies on the DA flow in NEC patients with congenital heart disease ${ }^{14,15)}$. In addition, our study results further support the proposed pathophysiology of NEC development, in which gut ischemia and injury with subsequent inflammatory reactions of the intestinal wall may play an important role ${ }^{4-7)}$. A decreased net forward flow along with an increased reverse flow of the DA (diastolic steal phenomenon) may be associated with multiple factors. A diastolic steal through a patent ductus arteriosus can cause significant diastolic reverse flow in the DA and may the compromise splanchnic circulation ${ }^{19,20)}$. However, in this study, there was no difference in the presence of HSDA between the NEC and control groups, indicating that other factors may play roles in causing diastolic reverse flow of the DA. Our previous study on neonates with hypoxic ischemic encephalopathy showed that substantial diastolic reverse flow was observed in the absence of patent ductus arteriosus ${ }^{17)}$. Preferential cerebral redistribution of cardiac output and increased peripheral resistance may explain the diastolic reverse flow and decreased blood flow to the $\mathrm{DA}^{17,18)}$. The significantly increased RI, a surrogate of peripheral vascular resistance, in the NEC group further supports the theory, although cerebral blood flow was not assessed in this study ${ }^{21)}$. Furthermore, the subgroup analysis of neonates with NEC according to NEC stages showed significant differences in the values of RI and diastolic reverse flow among the subgroups: the higher the NEC stage, the higher the increase in RI and diastolic reverse flow.

The strength of our study was that the hemodynamic parameters of NEC neonates were compared with those of a gestational age-matched control group. Concerning the basic clinical characteristics of the study population, there were no significant differences between the NEC and control groups in terms of gestational age, birth weight, Apgar scores, mode of delivery, breast feeding status, presence of umbilical arterial/venous catheter, and mode of invasive ventilator care. In addition, conventional left ventricular functional parameters including LVEF and LVFS were similar between the groups. Therefore, we conclude that altered blood flow patterns of the DA, decreased DABF along with increased peripheral vascular resistance, and diastolic reverse flow affect the splanchnic circulation, thereby leading to an increased risk of NEC development in preterm neonates. Logistic regression analysis also showed that an increasing RI of DA flow was an independent risk factor for the development of NEC.

This study has several limitations. As this was a retrospective, observational case-control study, the time intervals of echocardiographic study before the diagnosis of NEC were varied ( 0 to 7 days). Therefore, hemodynamic data obtained from a single echocardiographic study at different time points may not show a concrete association with the development of NEC. Thus, a well-scheduled serial echocardiographic study protocol to assess hemodynamic changes in preterm neonates may further elucidate the results of our study.

As the association between the flow patterns of the SMA and DA had not been clearly validated, changes in the flow characteristics of the DA do not necessarily mean an actual change in the SMA flow. However, we investigated the DABF pattern as a surrogate of the splanchnic circulation assuming that the DABF may have a direct influence on the SMA flow because the SMA is a major branch of the abdominal DA, and the sample point of the echocardiographic study in the DA was close to the origin of the SMA. Further studies are required to validate this issue. Nevertheless, we envisage that a blood flow study in the DA is easier and more feasible than that in the SMA in preterm babies, and can be a useful tool for stratifying in advance those neonates at a risk of developing NEC.

In preterm neonates with NEC, significant changes in the DABF characteristics were observed before the development 
of NEC compared with the control group. Decreased diastolic velocity and increased diastolic reverse flow along with increased peripheral vascular resistance resulted in decreased net forward flow in the DA, which affected the splanchnic circulation and thereby increased the risk of NEC development. Despite its limitations, a Doppler flow analysis in the DA may provide useful information on the hemodynamic changes in critically-ill preterm neonates, serve as a useful tool for the early stratification of preterm neonates at a risk of developing NEC, and ensure adequate management to improve outcomes in these patients.

\section{CONFLICT OF INTEREST}

No potential conflict of interest relevant to this article was reported.

\section{REFERENCES}

1) Mupsilonller MJ, Paul T, Seeliger S. Necrotizing enterocolitis in premature infants and newborns. J Neonatal Perinatal Med 2016;9:233-42.

2) Lim JC, Golden JM, Ford HR. Pathogenesis of neonatal necrotizing enterocolitis. Pediatr Surg Int 2015;31:509-18.

3) Schnabl KL, Van Aerde JE, Thomson AB, Clandinin MT. Necrotizing enterocolitis: a multifactorial disease with no cure. World J Gastroenterol 2008;14:2142-61.

4) Nowicki PT. Ischemia and necrotizing enterocolitis: where, when, and how. Semin Pediatr Surg 2005;14:152-8.

5) Chen Y, Chang KT, Lian DW, Lu H, Roy S, Laksmi NK, et al. The role of ischemia in necrotizing enterocolitis. J Pediatr Surg 2016; 51:1255-61.

6) Downard CD, Grant SN, Matheson PJ, Guillaume AW, Debski R, Fallat ME, et al. Altered intestinal microcirculation is the critical event in the development of necrotizing enterocolitis. J Pediatr Surg 2011;46:1023-8.

7) Gay AN, Lazar DA, Stoll B, Naik-Mathuria B, Mushin OP, Rodriguez MA, et al. Near-infrared spectroscopy measurement of abdominal tissue oxygenation is a useful indicator of intestinal blood flow and necrotizing enterocolitis in premature piglets. J Pediatr Surg 2011;46:1034-40.

8) Young CM, Kingma SD, Neu J. Ischemia-reperfusion and neonatal intestinal injury. J Pediatr 2011;158(2 Suppl):e25-8.

9) Zhang HY, Wang F, Feng JX. Intestinal microcirculatory dysfunction and neonatal necrotizing enterocolitis. Chin Med J (Engl) 2013;126:1771-8.
10) Hashem RH, Mansi YA, Almasah NS, Abdelghaffar S. Doppler ultrasound assessment of the splanchnic circulation in preterms with neonatal sepsis at risk for necrotizing enterocolitis. J Ultrasound 2017;20:59-67.

11) Murdoch EM, Sinha AK, Shanmugalingam ST, Smith GC, Kempley ST. Doppler flow velocimetry in the superior mesenteric artery on the first day of life in preterm infants and the risk of neonatal necrotizing enterocolitis. Pediatrics 2006;118:19992003.

12) Urboniene A, Palepsaitis A, Uktveris R, Barauskas V. Doppler flowmetry of the superior mesenteric artery and portal vein: impact for the early prediction of necrotizing enterocolitis in neonates. Pediatr Surg Int 2015;31:1061-6.

13) Fang S, Kempley ST, Gamsu HR. Prediction of early tolerance to enteral feeding in preterm infants by measurement of superior mesenteric artery blood flow velocity. Arch Dis Child Fetal Neonatal Ed 2001;85:F42-5.

14) Miller TA, Minich LL, Lambert LM, Joss-Moore L, Puchalski MD. Abnormal abdominal aorta hemodynamics are associated with necrotizing enterocolitis in infants with hypoplastic left heart syndrome. Pediatr Cardiol 2014;35:616-21.

15) Carlo WF, Kimball TR, Michelfelder EC, Border WL. Persistent diastolic flow reversal in abdominal aortic Doppler-flow profiles is associated with an increased risk of necrotizing enterocolitis in term infants with congenital heart disease. Pediatrics 2007; 119:330-5.

16) Walsh MC, Kliegman RM. Necrotizing enterocolitis: treatment based on staging criteria. Pediatr Clin North Am 1986;33:179201.

17) Yoon JH, Lee EJ, Yum SK, Moon CJ, Youn YA, Kwun YJ, et al. Impacts of therapeutic hypothermia on cardiovascular hemodynamics in newborns with hypoxic-ischemic encephalopathy: a case control study using echocardiography. J Matern Fetal Neonatal Med 2017 Jul 7 [Epub]. https://doi.org/10.1080/14767 058.2017.1338256.

18) Hochwald O, Jabr M, Osiovich H, Miller SP, McNamara PJ, Lavoie PM. Preferential cephalic redistribution of left ventricular cardiac output during therapeutic hypothermia for perinatal hypoxic-ischemic encephalopathy. J Pediatr 2014;164: 999-1004.e1.

19) Kessler U, Schulte F, Cholewa D, Nelle M, Schaefer SC, Klimek $\mathrm{PM}$, et al. Outcome in neonates with necrotizing enterocolitis and patent ductus arteriosus. World J Pediatr 2016;12:55-9.

20) Groves AM, Kuschel CA, Knight DB, Skinner JR. Does retrograde diastolic flow in the descending aorta signify impaired systemic perfusion in preterm infants? Pediatr Res 2008;63:89-94.

21) Bude RO, Rubin JM. Relationship between the resistive index and vascular compliance and resistance. Radiology 1999;211: 411-7. 